\title{
Characteristics and management of iatrogenic proximal humeral fracture during manual reduction of shoulder dislocation
}

\section{CURRENT STATUS: POSTED}

Research Square

Yan Zhou

Wuhan University Renmin Hospital

yanzhou0827@163.comCorresponding Author

ORCiD: https://orcid.org/0000-0003-1529-8746

Jianghua Ming

Wuhan University Renmin Hospital

Shiqing Liu

Wuhan University Renmin Hospital

DOI:

$10.21203 / \mathrm{rs} .2 .12548 / \mathrm{v} 2$

SUBJECT AREAS

Orthopedics

KEYWORDS

111 


\section{Abstract}

The authors have withdrawn this preprint from Research Square 